Apidologie, 1982, 13 (2), 143-155.

\title{
VARIATION EN FONCTION DE L'AGE DES OUVRIËRES D'ABEILLES (APIS MELLIFICA LIGUSTICA S.) DU POUVOIR D'ATTRACTION D'UN EXTRAIT DE PHÉROMONES ROYALES
}

\author{
Minh Hà PHAM, B. ROGER, Janine PAIN
}

I.N.R.A. - Station de Recherches sur l'A beille et les Insectes Sociaux, 91440 Bures sur Y'ette

\begin{abstract}
RÉSUMÉ
Le comportement de cour des ouvrières à l'égard d'une reine féconde a pu être reproduit au moyen de leurres. La substance responsable de l'attraction, non totalement identifiee dans sa fraction volatile, est localisée dans les glandes mandibulaires de la reine. La mise au point d'un test biologique appliqué à des lots d'ouvrières d'àge variable ( 1 à 30 jours) soumises à une même dilution de cet extrait ( $1 / 16$ tête de reine) permet de montrer une plus grande sensibilité des jeunes ouvrières de 1 à 6 jours à l'égard du leurre royal. L'existence d'une période " critique " au plan du comportement correspond aux données neurobiologiques concernant le développement du système olfactif.
\end{abstract}

\section{INTRODUCTION}

Depuis longtemps, on sait qu'une reine féconde attire un certain nombre d'ouvrières qui forment autour d'elle une " cour » qui se renouvelle constamment (Rösch, 1925 - TARANOv, IVANOVA, 1946).

Ce type de comportement a été étudié par deux approches différentes : d'une part, dans des conditions " naturelles " c'est-à-dire au niveau de la ruche, d'autre part, dans des conditions expérimentales strictement contrôlées, en laboratoire.

- Les tests en nature ont été utilisés par des auteurs tels que Gary (1961) qui isole des reines dans le haut des cadres d'une colonie. Mais cette méthode, selon VELTHUis et van Es (1964) permet de mesurer davantage l'agressivité des ouvrières à 
l'égard des reines que leur pouvoir attractif proprement dit. Ce test a été repris et modifié par Simpson (1979) avec un dispositif sur lequel sont déposés des extraits de reine.

- En laboratoire, l'influence de la reine a été étudiée par Voogd $(1956,1959)$ en utilisant différents types de leurres de reine.

BUTLER (1960) soumet des ouvrières à une expérience de choix entre ouvrières ou reines mortes et extraits de reine dans un olfactomètre.

VIERLING et RENNER (1977) évaluent le degré d'attraction d'extraits de reine par l'enregistrement du poids des abeilles attirées.

Les travaux de LAviE et PAIN (1959) ont permis de montrer que des glandes mandibulaires de reines attirent les ouvrières tout autant qu'une reine morte ou vivante. Le contenu de ces glandes inhibe d'autre part le développement des ovaires d'ouvrières orphelines (Butler, 1959) et la construction de cellules royales (ButLER, Simpson, 1958).

PaIN (1958) a dosé l'attraction exercée par des reines vivantes puis mis au point un test d'attraction (PAIN, 1961, PAIN et coll., 1962).

Or dès 1955, PaIN a observé que les jeunes ouvrières semblaient davantage attirées par des leurres de reines que les ouvrières les plus âgées.

L'ensemble de ces travaux nous ont conduit à nous interroger sur le rôle de la reine ou du substitut dans le déclenchement d'un comportement d'attraction des ouvrières et tout spécialement sur la mise en place et l'évolution de ce comportement au cours de la vie des ouvrières.

Il nous a semblé intéressant d'aborder l'étude de l'ontogénèse de ce comportement par le biais d'un test biologique, appliqué à des ouvrières de différentes classes d'âge. Ce test a pour but d'évaluer le degré d'attraction d'extrait de reines en fonction de l'âge des ouvrières testées, cette attraction étant définie au sens de PAIN (1961), comme un recrutement d'un certain nombre d'ouvrières et leur maintien à proximité immédiate de la reine ou du leurre.

Afin de contrôler strictement le paramètre " Age " des ouvrières, le biotest est effectué sur des lots d'abeilles encagées. Cette méthode facilite en outre le traitement statistique des résultats.

\section{MATÉRIEL ET MÉTHODE}

\section{A. - MATERIEL BIOCHIMIQUE}

\section{1 - Préparation de l'extrait attractif}

PaIN (1961) a défini la "substance royale " responsable de l'attraction de la reine, comme étant composée d'une Phéromone I (acide céto-9 décène- 2 oïque) et d'une Phéromone II, mélange de substances vola tiles non identifiées. Le mélange des deux reconstituant l'odeur de la reine. 
Nous avons observé que l'acide céto-9 décène- 2 oïque déposé sur un papier filtre $(8 \mu \mathrm{g})$ et présenté à de jeunes abeilles de 4 jours provoque le recrutement des abeilles mais pas leur cohésion autour du stimulus. Il n'y a donc pas d'attraction au sens où nous l'avons défini.

Ceci nous a amené à prendre en compte la substance royale dans son ensemble en préservant autant que possible les fractions volatiles lors de la préparation de l'extrait.

De plus, on sait que la substance attractive est principalement contenue dans les glandes mandibulaires des reines (BUTLER, 1959, BARBIER et coll., 1960). A la suite de ces différentes considérations, nous avons extrait à l'alcool éthylique un broyat de 500 têtes de reines conservees au congélateur. Nous avons chassé la totalité de l'éther par ébulition à $37^{\circ}$. L'effet comportemental de l'extrait ainsi obtenu est comparé à l'attraction exercée par une reine vivante.

Il apparait alors que l'extrait total entraine d'abord un effet répulsif avant de provoquer une attraction intense des ouvrières encagées. Afin d'atténuer cet effet, l'extrait a été dilué dans l'éther éthylique ( $2 \mathrm{ml})$ avant d'être scellé dans les ampoules de verre de $60 \mu$ l conservées au froid à $5{ }^{\circ} \mathrm{C}$.

Pour le test, l'extrait est déposé sur papier filtre (touche à parfum, FIRMENICH - Suisse) en prenant toutes les précautions nécessaires pour éviter une pollution de l'atmosphère environnant les cagettes (maintien de l'extrait au froid - Préparation des leurres dans un lieu distinct du lieu du déroulement des tests).

\section{2 - Analyse de l'extrait attractif par chromatographie en phase gazeuse.}

Les travaux de Pain, Hügel, Barbier (1960), de Callow, Chapman, Paton (1964), de Boch, SHEARER, Shuel (1979), ont permis d'identifier de nombreux composés dans les têtes et glandes mandibulaires de reines.

Notre analyse porte ici uniquement sur la teneur de l'extrait en acide céto-9 décène-2 trans oïque, composé principal du bouquet phéromonal de la reine. Nous avons utilisé un chromagaz à détection par ionisation de flamme (colonne inox $2 \mathrm{~m}$; diamètre : $1 / 4$ de pouce; phase : Kiselgur $80 / 100$ mesh imprégné de P.E.G. à $15 \%$ ).

Le chromatogramme ainsi obtenu permet de déterminer que, lors d'un test, chaque touche à parfum est imprégnée d'une dose d'extrait contenant $8,12 \mu \mathrm{g}$ d'acide céto- 9 décène- 2 oïque.

\section{B. - MATÉRIEL BIOLOGIQUE}

Les populations testées sont constituées d'abeilles de printemps. Elles proviennent de cadres de couvain naissant placés en étuve $\left(\mathrm{T}^{\circ} \mathrm{C}=33^{\circ} \mathrm{C}\right.$; Hygrométrie relative $\left.=50 \%\right)$. Le mode de prélèvement des ouvrières et leur répartition au hasard ont déjà èté décrits (Roger, PAIN, 1966). Les ouvrières âgées de quelques heures à 24 heures sont introduites dans des cagettes de type PaIN (PAIN, 1966). Lors des tests d'attraction, les parois normalement perforées sont remplacées par des vitres pleines afin de concentrer le stimulus.

Trente ouvrières sont introduites dans chaque cagette ce qui correspond au nombre minimal assurant à des populations d'abeilles encagées une durée de vie normale (Rinderer, BAXTER, 1978).

Les ouvrières encagées sont nourries selon la méthode décrite par ROGER (1971).

\section{C. $-M E E T H O D E S$}

Chaque lot d'abeilles n'est testé qu'une seule fois afin d'éviter tout phénomène de conditionnement aux odeurs. On connait en effet les capacités d'apprentissage des abeilles aux odeurs (von FRISCH, 1956).

Les abeilles testées sont réparties en 19 classes d'âges différents entre 1 jour et 30 jours. Ces différents lots d'abeilles sont testés dans un ordre aléatoire et non pas chronologique. Le vieillissement des abeilles a lieu en étuve. Pour chaque classe d'âge testée, l'observation est menée sur 10 cagettes parmi lesquelles on retient un témoin recevant un papier filtre imbibé de solvant seul. L'expérimentation se déroule à heure fixe et sa durée est limitée au maximum afin d'éliminer les effets du rythme circadien connus chez l'ouvrière (PAin, Roger, 1978). 
Les conditions expérimentales sont maintenues aussi stables que possible $\left(\mathrm{T}^{\circ} \mathrm{C}=22^{\circ} \mathrm{C} \pm 1{ }^{\circ} \mathrm{C}\right.$. Luminosité $=160 \mathrm{Lux}$ ). Au niveau des cagettes la luminosite est abaissée à 40 lux par un système de caches pour atténuer la perturbation causée aux abeilles par le passage de l'étuve obscure à la lumière artificielle.

On fixe à $20 \mathrm{mn}$ la durée d'un test d'attraction, pour une cagette donnée. C'est, en effet, le temps moyen nécessaire à l'établissement et à la stabilisation du phénomène d'attraction, lorsqu'il a lieu. Le nombre d'abeilles attirées est noté toutes les minutes. Le temps de latence qui sépare le début de l'expérimentation de la première manifestation comportementale significative (1 ${ }^{\text {re }}$ visite prolongée) n'est pas pris en compte.

Seul le nombre moyen d'abeilles attirées pendant la phase d'attraction maximale stable caractérise l'attraction. On appelle "Phase d'attraction maximale stable " la période durant laquelle le nombre des abeilles sur le leurre se stabilise aux valeurs les plus élevées de la période d'observation pendant au moins $3 \mathrm{mn}$.

La durée totale de ce plateau n'a pas été analysée car, pour certaines populations, la fin de l'attraction est intervenue après la fin de la période de test.

Ceci va dans le sens des travaux de Juska (1978) qui montrent un maintien du pouvoir attractif les 15 premières minutes, puis sa disparition après $45 \mathrm{mn}$ : au cours de ce test, une cage (grillagée, recouverte de cire, suspendue dans la colonie) ayant contenu une reine féconde vivante est pesée toutes les minutes pendant $45 \mathrm{mn}$. Le poids des ouvrières attirées par les traces phéromonées volatiles laissées par la reine donne une valeur relative de l'attraction et de sa durée.

\section{RÉSULTATS}

\section{A. - DÉTERMINATION D'UNE DOSE-SEUIL}

Afin d'établir une comparaison entre la réponse de populations d'abeilles d'âges différents, nous les avons toutes soumises à un stimulus identique.

L'extrait de départ ayant été obtenu à partir de 500 têtes, nous considérons que sa concentration est de 500 têtes-équivalents (T.E.). On effectue une gamme de dilutions (1 T.E. à 1/600 T.E.), chaque dilution étant testée à raison de $4 \mu 1$ sur chaque leurre.

Le nombre moyen d'abeilles attirées en fonction de la concentration (T.E.) du stimulus permet de tracer la courbe en coordonnées semi-logarithmiques (Fig. 1).

On constate que même à des concentrations très faibles (1/200 T.E.; 1/400 T.E.) l'extrait reste encore attractif. Seule la dose la plus diluée (1/600 T.E.) présente un pouvoir attractif très faible, proche de celui des témoins.

Aux doses intermédiaires $(1 / 64,1 / 32,1 / 16$ T.E.) le nombre d'abeilles attirées semble augmenter proportionnellement à la concentration de l'extrait jusqu'à la valeur $1 / 16$ T.E.

Au-delà, pour les doses les plus fortes $(1 / 8,1 / 4,1$ T.E.) une légère diminution de l'effet attractif apparait, ce qui pourrait traduire un effet répulsif des fortes concentrations.

L'analyse de ces résultats nous a conduit à fixer comme stimulus, la dose-seuil, définie comme la dose pour laquelle l'attraction est maximale pour une dilution minimale. 
Nombre moyen d'abeilles

attirées pendant la phose

d'attraction maximale stable

(échelle décimale)

15

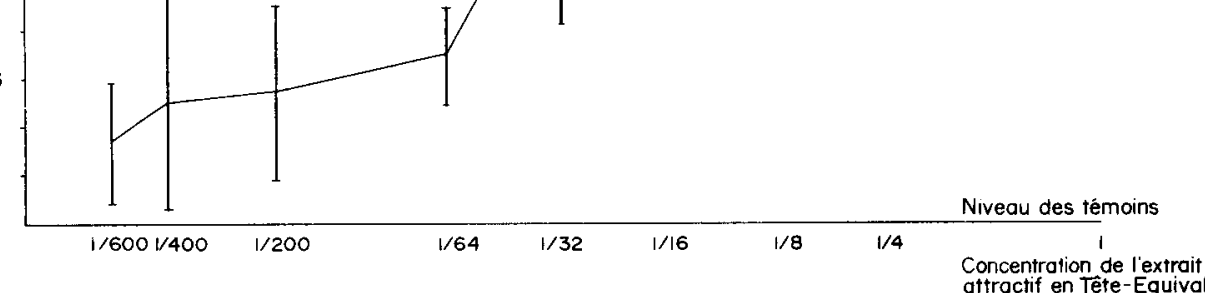

attroctif en Tête-Equivalent (Echelle logarithmique)

FiG. 1. - Attraction d'outrières d'abeilles (Apis mellifica ligustica $S$. ) en fonction de la concentration de l'extrait attractif.

FIG. 1. - Attractiveness of queen extract towards worker bees (Apis mellifica ligustica S.) versus the concentration of the extract.

$x$ axis : Mean number of bees attracted

during the "upper steady state of attraction" (Decimal scale).

$y$ axis : Concentration of extract

(in Queen head equivalent) (Logarithmic scale).

La dose $1 / 16$ T.E. (qui correspond à $8,12 \mu \mathrm{g}$ d'acide céto-9 décène-2 oïque) répond à cette définition et sera donc le stimulus utilisé tout au long de l'expérimentation.

\section{B. - BASES COMPORTEMENTALES DU TEST}

En préalable au test d'attraction, il est indispensable de faire l'analyse du comportement d'ouvrières placées en présence de l'extrait total attractif de reines.

L'observation porte sur 2 populations de 30 ouvrières âgées respectivement de 4 et 14 jours, afin de noter d'éventuelles modifications de comportement liées au vieillissement. 
On observe, pour les 2 populations, 2 phases comportementales :

Attraction à distance:

Les ouvrières, normalement placées dans les parties hautes de la cagette, s'orien tent vers la source de stimulus placée sur le sol, puis s'agrègent autour du site odorant.

Maintien des abeilles à proximité du leurre:

Sitôt le leurre localisé, les abeilles manifestent les postures caractéristiques suivantes :

- Exploration du leurre à l'aide de leurs extrémités antennaires.

- Succession de mouvements mandibulaires.

- Extension du proboscis et léchage du site odorant.

- Contacts tarsiques répétitifs avec le site qui peuvent correspondre à un marquage (Butler et coll., 1969; Chauvin, 1962; Lensky et coll., 1981).

- Nettoyage des antennes avec la première paire de pattes.

Les abeilles réagissent aux perturbations occasionnées par la mise en expérience à la fois par l'exposition de leur glande de Nasonov et par des vibrations des ailes. FreE (1968) interprète ce comportement rencontré chez les butineuses, comme un possible marquage d'une source de nourriture non odorante à l'intention de leurs congénères.

Collins (1981) remarque également ce comportement chez de jeunes ouvrières encagées soumises aux phéromones d'alarme. Le déclenchement de ce comportement n'est pas encore clairement compris.

Nous attendons le retour au calme des populations d'abeilles avant de mettre le test en route. Le principal critère retenu pour définir l'attraction est tout contact direct de l'ouvrière avec le leurre, que ce soit par ses antennes, ses pattes, ses pièces buccales ou son proboscis.

Les échanges trophallactiques, même effectués à proximité du leurre, ne sont pas pris en compte.

\section{C. - ANALYSE STATISTIQUE}

On effectue la moyenne des abeilles attirées pendant la phase d'attraction maximale stable pour chaque cagette puis pour chaque classe d'âge. Ces résultats permettent d'établir la courbe de la figure 2 qui représente, l'attraction des populations d'ouvrières testées, en fonction de leur âge.

\section{a) Analyse de la courbe}

Les nombres moyens d'abeilles attirées sont traités, en fonction des différentes classes d'âge, par la méthode du test $t$ de comparaison des moyennes. On constate 




Fug. 2. - Attraction d'ouvrières d'abeille (Apis mellifica ligustica $S$.) par un leurre phéromoné en fonction de leur âge (en jours).

Fig. 2. - Attractiveness of a pheromonal lure towards norker bees (Apis mellifica ligustica S.) rersus bee age (in days). $x$ axis : Mean number of bees attracted during the " upper steady state of attraction". $\mathrm{y}$ axis : Workerbees age.

d'une part que les populations testées présentent toujours une différence significative ou très significative par rapport aux groupes témoins chez lesquels l'attraction est nulle.

D'autre part, la courbe indique de façon très nette que l'attraction la plus importante apparait dans les populations âgées de 1 à 6 jours. Chez les ouvrières de 7 jours, l'attraction marque une chute. Pourles autres groupes, ce sont les ouvrières âgées de 10 et 24 jours qui réagissent le mieux.

L'importante différente entre les variances des populations testées, en particulier chez les populations âgées de 1 à 6 jours, perturbe la validité du test t. Pour y remédier, nous avons procédé à un regroupement des populations de plusieurs classes d'âge, afin d'établir l'histogramme de la figure 3.

b) Analyse de l'histogramme

Les populations sont regroupées en 5 super-classes d'âge allant de 6 jours en 6 jours, ce qui permet d'augmenter la taille de chaque échantillon. Les résultats obtenus permettent de confirmer la sensibilité particulière des jeunes ouvrières âgées de 1 à 


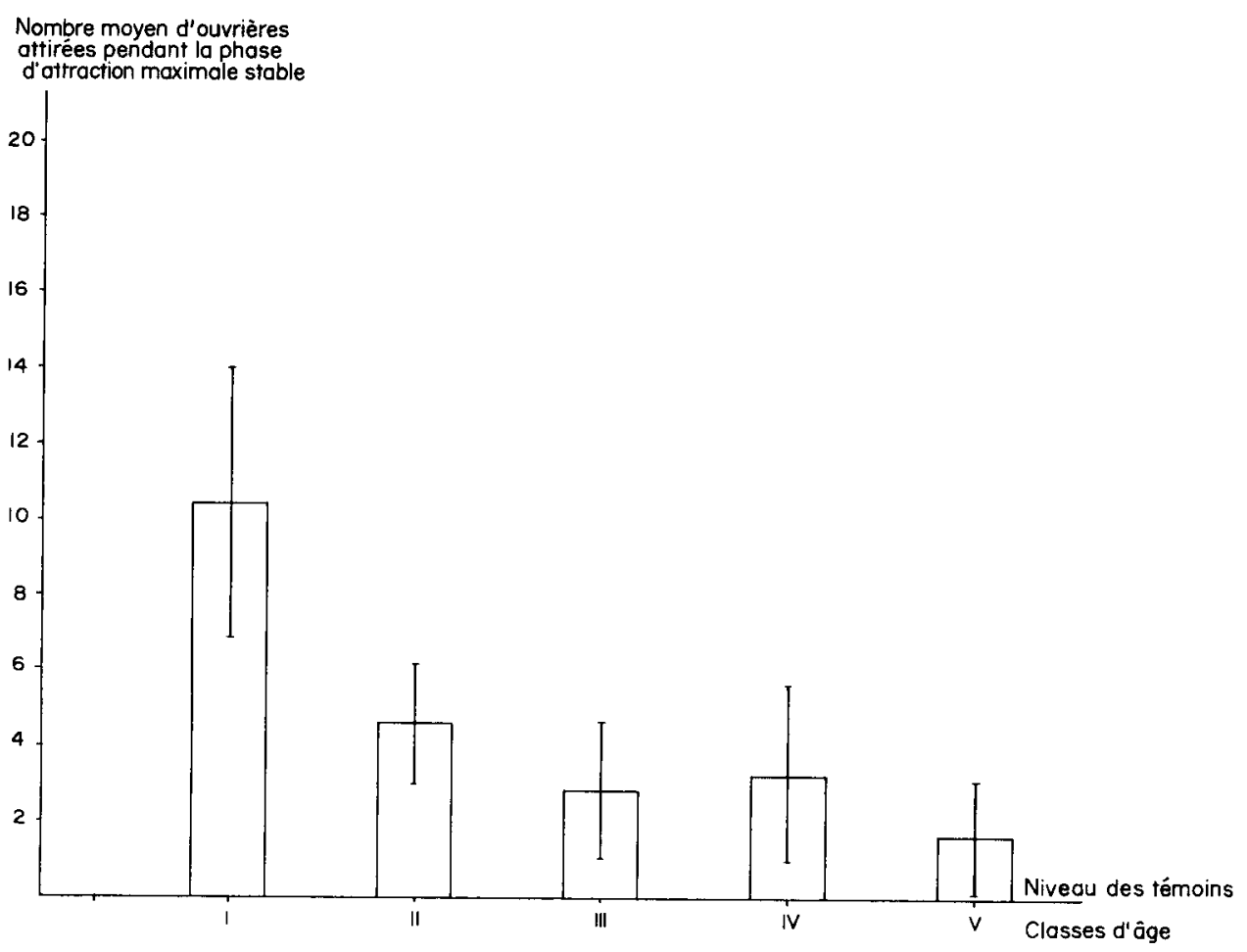

FIG. 3. - Attraction d'ouvrières d'abeille (Apis mellifica ligustica S.) par un leurre phéromoné en fonction de la classe d'âge.

Classe d'âge I : 1 à 6 jours.

Classe d'âge II : 7 à 12 jours.

Classe d'âge III : 14 à 18 jours.

Classe d'âge IV : 20 à 24 jours.

Classe d'âge $\mathrm{V}: 26$ à 30 jours.

FIG. 3. - Attractiveness of a pheromonal lure towards worker bees (Apis mellifica ligustica S.) versus age classes.

$x$ axis : Mean number of bees attracted during the "upper steady state of attraction". y axis : Age Classes.

Age class I : 1 to 6 days.

Age class II : 7 to 12 days.

Age class III : 14 to 18 days.

Age class IV : 20 to 24 days.

Age class $V: 26$ to 30 days.

6 jours en présence d'un leurre de reine. Cette réponse s'atténue au $7^{e}$ jour de vie avant de présenter une reprise chez les ouvrières de 10 jours puis une reprise moins marquée chez les ouvrières de 24 jours.

\section{D. - VÉRIFICATION DE LA STABILITÉ DE L'EXTRAIT}

A la fin de l'expérimentation, une série supplémentaire est testée pour vérifier si une dégradation de l'extrait attractif s'est produite malgré la conservation en ampoules 
scellées au froid. Cette série est constituée d'abeilles de 2 jours; cet âge a été retenu parce qu'il correspond à une des séries testée au tout début de l'expérimentation et qui donne la plus forte moyenne d'abeilles attirées par les leurres phéromonés.

La moyenne d'abeilles attirées dans cette $2^{\mathrm{e}}$ série n'est pas significativement différente de celle obtenue pour la $1^{\text {re }}$ série d'abeilles de 2 jours. On peut donc supposer que la substance attractive utilisée pour l'ensemble des tests ne s'est pas modifiée entre le début et la fin de l'expérimentation.

\section{DISCUSSION}

Les expériences qui viennent d'être décrites s'inscrivent dans le cadre d'une étude plus générale poursuivie dans notre laboratoire et concernant le problème de l'ontogénèse des comportements à déclencheurs chimiques chez l'abeille.

Du point de vue de leur sensibilité chimique, les abeilles sont considérées comme des insectes " généralistes " possédant simultanément 2 systèmes de détection des molécules chimiques (un système spécialiste et un système généraliste) (MAsson, 1980. Masson et Brossut, 1981).

Il se pourrait que chez les jeunes ouvrières âgées de 1 à 6 jours, le système adapté à la détection des odeurs phéromonales de la reine soit déjà fonctionnel. Par la suite, l'ouvrière devenant butineuse, le système de détection généraliste deviendrait simultanément plus performant.

L'analyse neurobiologique de la maturation post-natale du système olfactif d'ouvrières âgées de 1 à 21 jours indique déjà que la période comprise entre le $1^{\text {er }}$ et le $8^{\mathrm{e}}$ jour de vie imaginale est " critique ", aussi bien au niveau de la fonction (enregistrements électrophysiologiques globaux antennaires) qu'au niveau de la morphologie des neurorécepteurs (ARNOLD et MASSON, 1981).

Des expériences complémentaires en cours, prenant en compte d'une part le stimulus " phéromone royale ", d'autre part des stimuli " odeurs florales » devraient permettre de donner un début d'explication, sur le plan de la physiologie sensorielle aux expériences rapportées ci-dessus.

D'autre part, les ouvrières utilisées n'ayant jamais été au contact d'une reine ou de ses extraits jusqu'au moment de la présentation des leurres, on peut donc les considérer comme des abeilles naïves vis-à-vis de ce stimulus. La réponse observée ici suggère donc une acquisition des odeurs phéromonales royales pendant le développement préimaginal.

Les auteurs tiennent $\dot{a}$ remercier tout particulièrement $\mathrm{Cl}$. MASSON pour la lecture critique du manuscrit. 


\section{ZUSAMMENFASSUNG}

\section{VARIATION DER ATTRAKTIVITÄT EINES EXTRAKTES VON KÖNIGINNEN-PHEROMONEN IN BEZUG AUF DAS ALTER DER ARBEITSBIENEN (APIS MELLIFICA LIGUSTICA S.)}

Die Arbeitsbienen zeigen ein " Hofstaatverhalten " um eine begattete Königin oder um Duftattrappen einer Königin. Wir haben versucht, aus dem Ergebnis eines Biotests den Grad der Attraktivität von Pheromonextrakten von Bienenköniginnen auf Arbeitsbienen verschiedenen Alters zu bestimmen.

Die attraktive Substanz, die vor allem in den Mandibulardrüsen der Königin enthalten ist, besteht aus einer wenig flüchtigen Fraktion (Pheromon $1=$ trans-9-Oxo-2-decensäure) und einer Mischung flüchtiger, noch nicht zur Gänze bestimmter Fraktionen (Pheromon II). Es wurde ein Extrakt aus einem Homogenisat von 500 Königinnen-Köpfen in Äthyläther hergestellt.

Die Versuchstiere wurden in Gruppen von 30 Bienen in Käfigen vom Typ PAIN (1966) im Brutschrank $\left(33^{\circ} \mathrm{C}\right)$ gehalten. Die Gruppen wurden in 19 Altersstufen von 1 bis 30 Lebenstagen geteilt. Die Klassen wurden in zufälliger Reihenfolge als Proben von $10 \mathrm{Käfigen} \mathrm{pro} \mathrm{Klasse} \mathrm{getestet;} \mathrm{ein} \mathrm{Käfig}$ dieser Probe, versehen mit einer nur mit Lösungsmittel imprägnierten Attrappe, diente als Kontrolle.

Der Test wird charakterisiert durch die " Phase der stabilen Maximalattraktivität "; dies ist die Periode, während der - durch mindestens drei aufeinanderfolgenden Minuten - die Zahl der Bienen um die Attrape auf dem höchstem Niveau der ganzen Beobachtungszeit stabil bleibt.

In Vorversuchen konnte die Konzentration der Dosis des Extraktes für den Schwellenwert des Reizes bestimmt werden : Er entspricht 1/16 des Kopfes einer Königin, mit einem Gehalt von 8,12 $\mu \mathrm{g}$ trans-9Oxo-2-decensäure. Für diese ist die Attraktivität maximal bei niedrigster Verdünnung (Fig. 1).

Bei Vorhandensein der Attrappe ( $4 \mu 1$ Extrakt von der Schwellendosis, auf einem Parfumtupfer), beobachtet man das Phänomen der Attraktion auf Distanz und dann ein Verharren der Arbeiterinnen in der Nähe der Attrappe. Das für die Bewertung der Attraktivität benutzte Kriterium ist der direkte Kontakt der Arbeiterinnen mit der Attrappe (mittels der Antennen. Beine oder Mundwerkzeugen).

Auf Figur 2 ist die mittlere Zahl angelockter Bienen in Abhängigkeit von ihrem Alter eingezeichnet. Die statistische Analyse dieser Daten (Vergleich der Mittelwerte mit dem t-Test) ergibt einerseits eine signifikante Differenz des Verhaltens zu den Kontrollgruppen, andererseits eine stärkere Attraktivität auf die Altersgruppen zwischen 1-6 Lebenstagen und einen Abfall der Attraktion für Altersstufen über 7 Tagen. Diese Resultate erfahren durch Gruppierung der Daten in 5 Superklassen mit Altersstufen von 6 zu 6 Tagen eine Bestätigung (Fig. 3).

Die Stabilität des Extraktes in Hinblick auf das Verhalten wurde am Ende der Versuchsperiode überprüft.

Diese Periode zwischen dem 1. und 6. Lebenstag, in der Jungbienen gegenüber der PheromonKöniginattrappe besonders empfindlich zu sein scheinen, entspricht einer kritischen Periode der Entwicklung des Geruchssinnes, sowohl auf der Ebene der Funktion wie auf der Ebene der Struktur der Neurorezeptoren. Gegenwärtig werden diese Ergebnisse auf dem Gebiet der Sinnesphysiologie weiterverfolgt.

\section{SUMMARY}

\section{ATTRACTIVENESS OF A QUEEN PHEROMONAL EXTRACT TO WORKER BEES OF VARYING AGES (APIS MELLIFICA LIGUSTICA S.)}

The " courtship behaviour " of worker bees towards a fertile queen can be reproduced using olfactive lures. By the mean of bioassay, the attractiveness of queen pheromonal extracts can be evaluated to worker bees of varying ages.

The attracting substance, mainly found in queen mandibular glands, is composed of a slightly volatile fraction (Pheromone I : 9-oxodec-2 enoic acid) and a mixture of not completely identified volatiles (Pheromone II). An extract was prepared from 500 queen heads in ethyl ether. 
Test bees were held in an incubator $\left(33^{\circ} \mathrm{C}\right)$ in groups of 30 bees, per PaIN's type cage. The groups were divided in 19 age classes from 1 to 30 days. The different classes were tested at random using a 10 cage sample for each age class, with one solvent control. The test was characterized by the "upper steady state of attraction " which was the highest number of bees on the lure during the 3 mn observation period.

Preliminary experiments indicated a threshold concentration of the stimulus (corresponding to $1 / 16$ queen head and containing 8,12 $1 / \mathrm{g}$ 9-Oxodec-2 enoic acid), which gave the highest attraction for the lowest dilution (Fig. 1).

In the presence of the lure $(4, \mu$ ) of extract at its threshold level on filter paper), we observed a phenomenon of long range attraction and stabilization of worker bees near the lure. The criterion adopted to define attractiveness was direct contact with the lure (by antennae, legs or mouth parts).

The mean number of bees attracted and their ages, are shown in figure 2. The statistical analysis of these data showed a significant difference in reponses towards controls and more important, attractiveness in populations of bees 1 to 6 days old and a decrease from the 7th day. These results are corroborated by a statistical treatment applied to 5 age classes increasing by 6 days (Fig. 3). The stability of the extract from the behavioural point of view was examined at the end of the experiment.

This age of 1 to 6 days, while the young bees seem particularly sensitive to a queen pheromonal lure, would correspond with a critical period in olfactive development both on the level of function and structure of neuroreceptors.

We are now conducting further experiments in the field of sensory physiology.

\section{BIBLIOGRAPHIE}

Arnold G., Masson C., 1981. - Évolution, en fonction de lâge, de la structure externe des sensilles olfactives de l'antenne de l'ouvrière d'abeille A pis mellifera L. C.R. Acad. Sci. Paris, ser. 3, 292, 681 . 686.

Barbier M., Pain J., 1960. - Étude de la sécrétion des glandes mandibulaires des reines et des ouvrières d'abeilles (Apis mellifica L.) par chromatographie en phase gazeuse. C.R. Acad. Sci., Paris, 250, 3740-3742.

Boch R., Shearer D. A., Shuel R. W., 1979. - Octanoic and other volatile acids in the mandibular glands of the honeybee and in royal jelly $J$. Apic. Res., 18 (4), 250-252.

Butler C. G., 1959. - The source of the substance produced by a queen honeybee (Apis mellifica L.) which inhibits development of the ovaries of the workers of her colony. Proc. R. Ent. Soc. Lond. (A). 34, $137-138$.

Butler C. G., 1960. - Queen Recognition by Worker Honeybees (Apis mellifica L.). Experientia, 16 (9), 424-428.

Butler C. G., et al., 1969. - Nest entrance marking with pheromones by the honeybee Apis mellifera L. and by a wasp, Vespula vulgaris L. Anim. Behav., 17, 142-147.

Butler C. G., Simpson J., 1958. - The source of the queen substance of the honeybee (Apis mellifica L.). Proc. R. Ent. Soc. Lond. (A), 33, 120-122.

Callow R. K., Chapman J. R., Paton P. N., 1064. - Pheromones of the honeybee : chemical studies of the mandibular gland secretion of the queen. J. apic. Res.; 3 (2), 77-89.

Chauvin R., 1962. - Sur l'épagine $\varepsilon$ et sur les glandes tarsales d'Anhart. Insectes sociaux, 9, 1, 1-5.

Collins A. M., 1981. - Effects of temperature and humidity on honeybee response to alarm pheromones. J. apic. Res., 20, 1, 13-18.

FreE J. B., 1968. - The conditions under which foraging honeybees expose their Nasanov glands. J. apic. Res., 7 (3), 139-145. 
FRISCH von K., 1956. - Lernvermögen und erbgebundene Tradition im Leben der Bienen. In : l'instinct dans le comportement des animaux et de l'homme, Paris, Masson, 1956, 345-386.

GARY N. E., 1961. - Queen honeybee attractiveness as related to mandibular gland secretion. Science, $133,1479-1480$.

JUSKA A., 1978. - Temporal decline in attractiveness of honeybee queen tracks. Nature, 276, $\mathrm{n}^{\circ} 5685$, 261-262.

Lavie P., Pain J., 1959. - Relation entre la substance attractive, le facteur antibiotique et le développement ovarien chez la reine d'abeille. C. R. Acad. Sci., Paris, 248, 3753-3755.

LENSKY Y., SLABerzKI Y., 1981. - The inhibiting effect of the queen bee (Apis mellifera L.) foot-print pheromone on the construction of swarming queen cups. J. Insect. Physiol., 27 (5), 313-323.

Masson C., 1980. - Mécanismes de détection et d'identification des phéromones par le système nerveux de l'insecte. C.R. Colloque int : "Phéromones sexuelles et Médiateurs chimiques chez les Insectes", Colmar Nov. 1980 (publ. 1981 11-18).

Masson et Brossut R., 1981. - La communication chimique des insectes. La Recherche, $\mathrm{n}^{\circ} \mathbf{1 2 1}, 406$ 416.

PaIN J., 1955. - Dosage biologique et spectographie de l'ectohormone des reines d'abeilles. V.R. Acad. Sci., Paris, 240, 670-672.

PaIN J., 1958. - Mesure du pouvoir inhibiteur et de l'attractivité de l'ectohormone des reines d'abeilles. Différences individuelles. C.R. Acad. Sci., Paris, 242, 1080-1082.

Pain I., 1961. - Sur la phéromone des reines d'abeilles et ses effets physiologiques. Ann. Abeille, 4 (2), 162 pages.

PaIN J., 1966. - Nouveau modèle de cagettes expérimentales pour le maintien d'abeilles en captivité. Ann. Abeille, 9 (1), 71-76.

Pain J., Hügel M. F., Barbier M., 1960. - Sur les constituants du mélange attractif des glandes mandibulaires des reines d'abeilles (Apis mellifica L.) à différents stades de leur vie. C.R. Acad. Sci, Paris, 251, 1046-1048.

PAIN J. et al., 1962. - Chemistry and biological activity of the secretions of queen and worker honeybees (Apis mellifica L.). Comp. Biochem. Physiol., 6, 233-241.

PAIN J., Roger B., 1978. - Rythme circadien des acides cèto-9 décène- 2 oïque phéromone de la reine et hydroxy-10 décène-2 oïque des ouvrières d'abeilles Apis mellifica ligustica S. Apidologie, 9 (4), 263 272.

RINDERER T. E., BAXTER J. R., 1978. - Honeybees : The effect of group size on longevity and hoarding in laboratory cages. Am. Entomol. Soc. Amer., 71 (5), 732.

Roger B., 1971. - L'influence de la reine d'abeille (Apis mellifica L.) sur la prise de nourriture des ouvrières accompagnatrices. Apidologie, 2 (2), 123-155.

Roger B., PAIN J., 1966. - L'influence de la reine d'abeille (Apis mellifica L.) sur le taux de mortalité des ouvrières accompagnatrices. Am. Abeille, 9 (1), 5-36.

RösCH G. A., 1925. - Untersuchungen über die Arbeitsteilung im Bienenstaat I. Die Tätigkeiten im normalen Bienenstaate und ihre Beziehungen zum Alter der Arbeitsbienen. Z. Vergl. Physiol., 2, 571 . 631.

Shaposhnikova N. G., Gavrilov B. N., 1973. - On perception by worker bees (Apis mellifera caucasica C.) of synthetic pheromone of the queen substance (9-oxodec-trans-2-enoic acid). En russe. Zool. $Z h$, 52 (2), 291-293.

SiMPSON J., 1979. - The existence and physical properties of pheromones by which worker honeybees recognize queens. J. apic. Res., 18 (4), 233-249.

Taranov G. F., Ivanova L. V., 1946. - Observations upon queen behaviour in bee colonies. En russe. Pchelovodstvo, 2 (3), 35-39.

Velthuis H. H. W., Van Es J., 1964. - Some functional aspects of the mandibular glands of the queen honeybee. J. apic. Res., 3 (1), 11-16. 
Vierling G., RenNer M., 1977. - Die Bedeutung des Sekretes der Tergittaschendrüsen für die Attraktivität der Bienenkönigin gegenüber jungen Arbeiterinnen. Behav. Ecol. Socio. biol., 2, 185200 .

Voogd S., 1956. - The Influence of a Queen on the Ovary Development in Worker Bees. Experientia, $12(5), 199-201$.

Voogo S., 1959. - How worker Bees perceive the presence of their queen. Z. vergl. Physiol., 41, 527-582. 